\title{
APROXIMACIÓN DE UNA PROPUESTA METODOLÓGICA PARA EL EMPRENDIMIENTO E IMPLEMENTACIÓN DE LA INNOVACIÓN EN PYMES
}

Approximation of a methodological proposal for the entrepreneurship and implementation of innovation in SMEs / Aproximação de uma proposta metodológica para o empreendimento e implementação da inovação em PYMES

Israel Cordero Beltrán ${ }^{1}$, Andrés Valdéz Zepeda ${ }^{2}$ y Cristina Lizeth Ramírez Cuevas ${ }^{3}$

\section{RESUMO}

A inovação constitui, para as empresas, um papel importante, sendo que a sua correcta aplicação poderá contribuir para que estas sejam mais competitivas e produtivas. A presente investigação tem como objectivo a análise conceptual da inovação, os tipos e modelos aplicáveis, com a finalidade de identificar, por um lado, os seus pontos convergentes e, por outro lado, poder facilitar o empreendimento, no que concerne aos processos de inovação em PYMES. A investigação foi realizada mediante a análise de diferentes artigos e livros (García e Rodríguez, 2010; Kline, e Rosenberg, 1986; Marquis, 1969; Rothwell, 1994; Rothwell, e Zegveld, 1985), cujo objectivo é compreender como se dá a inovação e tudo o que se necesita, para uma aplicação eficiente. Os resultados obtidos revelam que há necessidade de identificar ferramentas que facilitem a implementação de um processo de inovação, especialmente nas PYMES, devido à insuficiência de recursos que estas empresas apresentam e que, ao mesmo tempo, ajudem a melhorar a compreensão a cerca do fenómeno de inovação.

Palavras Chave: empreendimento, pymes, modelos de inovação, ferramentas, implementação.

Recebido: Novembro 2018

\section{Aceitado: Dezembro 2018}

\section{RESUMEN}

La innovación constituye para las empresas un papel importante debido a que su correcta aplicación puede contribuir para que éstas sean más competitivas y productivas. La presente investigación ha tenido como objetivo el análisis conceptual de la innovación, los tipos y modelos aplicables con la finalidad de identificar en que coinciden y poder facilitar el emprendimiento con respecto a los

\footnotetext{
${ }^{1}$ Doctor en Ciencia del Comportamiento, docente en la Universidad de Guadalajara y Docente investigador en la Universidad UTEG. Guadalajara, México. Correo electronico: israel.cordero@uteg.edu.mx / icorderob@cucea.udg.mx

2 Doctor en Estudios Latinoamericanos con especialidad en Ciencias Políticas, Jefe del Departamento de Administración en el Centro Universitario de Ciencias Económico-Administrativas, Universidad de Guadalajara. México. Correo electronico: azepeda@cucea.udg.mx

${ }^{3}$ Licenciatura en Administración de Empresas, Centro Universitario de Ciencias EconómicoAdministrativas, Universidad de Guadalajara, Núcleo Universitarios Los Belenes, Zapopan, Jalisco, México.
} 
procesos de innovación en PYMES. La investigación fue realizada mediante el análisis de diferentes artículos y libros (García \& Rodríguez, 2010; Kline, \& Rosenberg, 1986; Marquis, 1969; Rothwell, 1994; Rothwell, \& Zegveld, 1985) con el objetivo de comprender cómo se da la innovación y todo lo que se necesita para una aplicación eficiente. Los resultados obtenidos apuntan a la necesidad de identificar herramientas que faciliten la implementación de un proceso de innovación, especialmente en las PYMES debido a la carencia de recursos que estas empresas presentan, y que al mismo tiempo ayuden a la mejor comprensión del fenómeno de innovación.

Palabras clave: emprendimiento, PYMES, modelos de innovación, herramientas, implementación.

\begin{abstract}
Innovation is an important role for companies because their correct application can contribute to make them more competitive and productive. The present research has had as object the conceptual analysis of the innovation, the types and applicable models with the purpose of identifying in which they agree and to facilitate the creation of new opportunities with respect to the processes of innovation in SMEs. The research was carried out through the analysis of different articles and books (García \& Rodríguez, 2010; Kline, \& Rosenberg, 1986; Marquis, 1969; Rothwell, 1994; Rothwell, \& Zegveld, 1985), with the aim of understanding how innovation occurs and all that is needed for an efficient application. The results pointed to the need to identify tools that facilitate the implementation of an innovation process, especially in small and medium enterprises (SMEs) due to the lack of resources that these companies present and that at the same time help the best understanding of the phenomenon of innovation.
\end{abstract}

Key words: entrepreneurship, SMEs, innovation models, tools and implementation.

\title{
INTRODUCCIÓN
}

La globalización de los mercados y la economía, demandan de las empresas un esfuerzo innovador, mismo que debe ser apoyado por todas las organizaciones que conforman el sistema económico (Aranda, De la Fuente, \& Becerra, 2010).

La innovación constituye un papel importante dentro de las pequeñas y medianas empresas (PYMES) debido a que estas representan el segmento de mayor aporte a la economía del país siendo el $98.6 \%$ del total de las entidades económicas de México (Instituto Nacional de Estadística y Geografía [INEGI], 2014). La necesidad de innovar surge de la alta competencia que existe entre este tipo de empresas y tiene por objetivo posicionarlas en el mercado. La innovación otorga a las empresas la posibilidad de captar una mayor cantidad de clientes, ser más 
Aproximación de una propuesta metodológica para el emprendimiento e implementación de la innovación en PYMES

competitivas y productivas debido a que captan nuevas oportunidades en el mercado y con esto lograr convertirse en líderes del mercado (Ortíz, 1994; Porter, 1998).

Las PYMES se caracterizan por tener poca disposición para implementar procesos de innovación debido a que demandan diversos tipos de inversiones, entre ellas la formación de recursos humanos, así como la modernización de la capacidad tecnológica y organizativa (Genatios \& Lafuente, 2004). Por lo que los cambios que se realizan, generalmente provienen del deseo o necesidad del cliente (Pedroza \& Sánchez 2005).

El continuo cambio en los mercados ha provocado que las empresas busquen emplear nuevas metodologías de innovación en el menor tiempo posible (Martín, 1995) y la competencia global es uno de los factores estimulantes en la implementación de innovación en las PYMES (Heijs, 2001) junto con los individuos que forman parte importante para innovar debido a que puede adoptar mejores prácticas y técnicas (Schumpeter, 1967).

Un paso previo a la implementación de la innovación en las organizaciones, especialmente en las PYMES, es la identificación de las barreras que existen, dificultan y perjudican la implementación de la misma. Estas varían según el tipo de actividad que la empresa realice y el grado de innovación que se tenga actualmente, y se pueden clasificar como internas (i.e., rigidez en la cultura empresarial, escasos fondos internos, cuestiones en recursos humanos y sistemas contables) y externas a la organización (i.e., factores relacionados con la oferta y demanda, acceso a recursos financieros, materias primas o información tecnológica, así como cuestiones políticas) (García \& Martínez, 2009).

En las PYMES, el tamaño y la escasez de diferentes recursos representan una limitante para acceder a la innovación (Heijs, 2001). A menudo carecen de recursos financieros propios y suficientes para realizar proyectos de innovación (Freel, 1998; Organización para la Cooperación y el Desarrollo Económicos [OCDE], 2006; Fernández \& Rodeiro-Pazos, 2006; Zan, 2000) y tienen mayor dificultad para obtener un financiamiento en comparación con las grandes empresas (OCDE, 2006) además de que no cuentan con recursos humanos técnicamente calificados 
(Moreno, 2013; Fernández \& Rodeiro-Pazos, 2006; Zan, 2000) y pueden llegar a presentar deficiencias en otras áreas como administración y mercadotecnia (Freel, 1998).

Por tanto el presente documento tiene como objetivo el realizar un análisis conceptual de la innovación, los tipos y modelos aplicables, para a través de esto poder identificar semejanzas que faciliten el emprendimiento con respecto a los procesos de innovación en PYMES mediante una posible propuesta metodológica. Así mismo, la investigación fue realizada bajo un nivel descriptivo y con un enfoque cualitativo, mediante la recolección de datos obtenidos de artículos científicos y libros, con la finalidad de comprender mejor el fenómeno de la innovación y las diversas herramientas que pueden ser de utilidad en el proceso de la misma y por consiguiente presentar la propuesta metodológica. De esta manera el documento se organiza comenzando por conceptualizar a la innovación, sus tipos y un análisis de los modelos y sus semejanzas, para posteriormente dar paso a la propuesta y las conclusiones de este documento.

\section{CONCEPTUALIZACIÓN DE LA INNOVACIÓN}

Diversos autores han realizado aportes al concepto de innovación y han identificado diferentes factores y herramientas que intervienen para su aplicación. Se puede definir la innovación como la combinación de conocimientos cuya aplicación es útil para resolver un problema o atender una necesidad (Solleiro \& Castañon, 2010; Tundidor, 2017). La innovación ayuda a detectar nuevos productos, procesos, servicios, métodos de comercialización o métodos organizativos (Aranda, De la Fuente, \& Becerra, 2017; OCDE, 2006) y adquiere importancia cuando su aplicación se traduce en valor agregado (García, \& Rodríguez, 2010).

Hay innovaciones que dependen de la tecnología, pero también existen innovaciones sin connotación tecnológica, sobre todo en los servicios (Battini, 1994) lo que está claro es que la innovación implica generar un cambio en la forma de realizar las cosas (Escorsa \& Valls, 2005; Pedroza, Garrido, López, Ortiz, \& Ortiz, 2013; Tundidor, 2017). 
Aproximación de una propuesta metodológica para el emprendimiento e implementación de la innovación en PYMES

Al hablar de los beneficios de la innovación se identifica a dos partes, en el ámbito empresarial, la implementación de la innovación traerá consigo reducción de costos e incremento de la calidad y para los usuarios finales, la innovación se refleja en mejores productos o servicios (Solleiro \& Castañon, 2010).

La innovación puede darse de dos maneras: radical e incremental, esto dependiendo de las necesidades que tenga la organización. La innovación radical genera un nuevo conocimiento volviendo obsoletos los que se utilizan actualmente (Afuach, 1999; García \& Rodríguez, 2010) y genera como resultado un nuevo producto o un nuevo proceso (Fernández, 1996). Mientras que la innovación incremental se presenta en una organización mediante la introducción paulatina de mejoras en productos y procesos (García \& Rodríguez, 2010) o la mejora de los componentes de un producto sin alterar la relación entre ellos (Fernández, 1996) y evitando la obsolescencia (Afuach, 1999). Su principal característica es la corta duración, debido a que proviene del ingenio natural de una persona y suele ser aplicable a la resolución de un problema, por lo que en el corto plazo puede presentarse una situación en la que alguien pueda mejorar cualquier idea (Wasserman, 2012).

\section{TIPOS DE INNOVACIÓN}

La OCDE (2006) identifica cuatro tipos de innovaciones: de producto, de proceso, en marketing y organizativa, que pretenden mantener una continuidad con respecto de la definición de innovación. En conjunto con esto, diferentes autores (Escorsa \& Valls, 2003; Fernández, 1995; Velázquez, \& Salgado, 2016) coinciden en la existencia de un tipo de innovación adicional a los ya mencionados, denominado innovación tecnológica.

La innovación será tecnológica cuando se involucre conocimiento científico y tecnología, al conjunto de actividades organizacionales, financieras y comerciales que se aplican en un proceso de producción de nuevos bienes y servicios, realizando cambios tecnológicos y haciendo uso del conocimiento de las necesidades del mercado de una forma rentable para la empresa (Escorsa, \& Valls, 2003; Fernández, 1995; Velázquez, \& Salgado, 2016). 
La innovación en productos se da con la introducción de un bien o servicio completamente nuevo o, significativamente mejorado y diferente de los productos actuales con que cuente la empresa, haciendo uso de nuevos conocimientos y tecnología (OCDE, 2006). La mejora realizada deberá estar enfocada hacia las características o el uso para el cual fue diseñado el producto (García \& Rodríguez, 2010) por lo que se deberán implementar cambios en las materias primas con la finalidad de tener un mejor rendimiento (OCDE, 2006; Schumpeter, 1934).

Es importante mencionar que la OCDE (2006) refiere que la palabra producto involucra los bienes y servicios al mismo tiempo. Por el contrario, Schumpeter (1934) al hablar de innovación en productos hace referencia únicamente a la introducción de nuevo bien o una nueva clase de bienes sin incluir el giro de los servicios.

La innovación en procesos consiste en implementar un nuevo, o significativamente mejorado proceso de producción o distribución que no haya sido utilizado con anterioridad en la empresa (OCDE, 2006; Schumpeter, 1934) con la finalidad de disminuir los costos e incorporar una mejora de calidad en los procesos (OCDE, 2006) teniendo en cuenta que no pueden utilizarse métodos de producción actuales o convencionales (Dinacyt, 2003).

Para la innovación en marketing es necesaria la aplicación de un nuevo método de comercialización diferente a los utilizados anteriormente por la empresa y realizando a su vez, cambios significativos en el diseño, envasado o promoción del producto, con la finalidad de satisfacer a los consumidores, tener apertura de nuevos mercados y posicionar el producto de forma diferente en el mercado (OCDE, 2006).

La innovación organizativa o de tipo organizacional hace referencia a la capacidad de crear nuevos conceptos de negocio completamente distintos a los ya existentes en las organizaciones (Hamel, 2001) y se relaciona con la interacción existente entre las diferentes áreas dentro de la organización y la relación de la organización misma con otras empresas que se encuentran en su entorno (Comisión Europea, 1995; OCDE, 2006). Su principal objetivo es organizar rutinas y procedimientos dentro de la empresa mediante nuevos diseños organizacionales 
Aproximación de una propuesta metodológica para el emprendimiento e implementación de la innovación en PYMES

(Arraut, 2008; OCDE, 2006) y su implementación deberá ser una decisión tomada por la dirección de la empresa (OCDE, 2006).

\section{MODELOS DE INNOVACIÓN}

Diversos autores (Kline \& Rosenberg, 1986; Marquis, 1969; Rothwell, 1994; Rothwell \& Zegveld, 1985) proponen una serie de modelos aplicables a la innovación:

Los modelos de empuje de la tecnología (technology push) de Rothwell (1994) tienen como base el conocimiento científico, seguido de la ingeniería y diseño, producción, marketing y ventas. García, \& Rodríguez (2010) señalan que hacer un modelo lineal, sería imposible que la dinámica innovadora comenzará en cualquier otra parte del modelo que no fuera la investigación.

En los modelos de tirón de la demanda de Rothwell (1994) se muestran las necesidades el mercado como la base, siguiendo con el desarrollo, producción y ventas. García, \& Rodríguez (2010) señalan la importancia de conocer las necesidades de los consumidores debido a que son una fuente de ideas para el departamento de I+D.

Los modelos interactivos (coupling models) de Rothwell, \& Zegveld (1985) parten de las nuevas necesidades y la nueva tecnología para generar ideas, mismas que pasaran por un proceso de investigación, diseño y desarrollo para ser producidos y vendidos. Para García, \& Rodríguez, (2010) estos modelos pretenden explicar la influencia del mercado en la innovación, misma que aporta conocimientos con respecto a las necesidades de los clientes y las fuerzas tecnológicas, mismas que aportan nuevas técnicas de producción, y esto en conjunto facilita la creación de nuevas ideas.

El modelo de Marquis (1969) parte de dos situaciones, la primera es el estado de los conocimientos técnicos y la segunda es el entorno económico y social, seguido de esto se deberá reconocer una demanda potencial y tener factibilidad técnica para generar una idea, darle seguimiento en el departamento de I+D, generar una solución, implementarla y darle difusión. García, \& Rodríguez, (2010) explican que en este modelo las ideas pueden venir de distintos departamentos (i.e., 
producción, comercialización, logística) no necesariamente del departamento de investigación.

En el modelo de innovación de enlaces de cadena de Kline \& Rosenberg, (1986) se parte de la investigación y desarrollo y los conocimientos científicos disponibles, para seguir con una serie de actividades relacionadas con el mercado potencial, la invención de un diseño, detallado de pruebas, producción y finalmente la distribución y comercialización. García \& Rodríguez, (2010) señalan en que en este modelo las actividades realizadas se relacionan unas con otras y generan un resultado incierto, además, la I+D no se presenta como una fuente de invención, sino como una herramienta para solucionar problemas dentro del proceso de innovación.

\section{PROPUESTA METODOLÓGICA}

Con base en la información obtenida de los modelos de innovación (García \& Rodríguez, 2010; Kline, \& Rosenberg, 1986; Marquis, 1969; Rothwell, 1994; Rothwell, \& Zegveld, 1985), se determina que estos coinciden en la necesidad de realizar investigación científica o algún tipo de investigación que ayude a conocer las necesidades del mercado, plantear un diseño del producto que cumpla con las especificaciones y necesidades de la demanda y generar una retroalimentación que complemente el proceso de innovación y al mismo tiempo genere nuevas ideas para futuras innovaciones que pudieran implementarse en la empresa.

Aunque los modelos tienden a explicar el proceso para llevar a cabo la innovación, presentan como deficiencia el hecho de que no cuentan con herramientas específicas que faciliten su implementación, específicamente en estas tres etapas o actividades (i.e., investigación científica y/o de las tendencias del mercado, diseño del producto y retroalimentación) en las que los autores coinciden y que se podrían definir como las más importantes o sobresaliente dentro del proceso por lo que complica aún más la situación para una PYME que desee implementar algún tipo de innovación.

Con base en lo analizado en los modelos de innovación así como las limitantes que las PYME enfrentan, se considera necesario identificar una serie de 
Aproximación de una propuesta metodológica para el emprendimiento e implementación de la innovación en PYMES

herramientas que faciliten el proceso de innovación, específicamente para estas empresas, por lo que se plantea la siguiente propuesta.

Principalmente deberá investigarse el mercado con respecto de los competidores, lo que puede ser mediante la herramienta benchmarking debido a que diferentes autores (Franco de Abreu, Giuliani, Kassouf \& Alves, 2006; Intxaurburu \& Velasco, 2010; Martín, 1995) lo proponen como herramienta de innovación y mejora continua aplicable a cualquier tipo de organización, analizando los factores críticos de competitividad o benchmarks, tales como el precio, diseño, distribución, tiempos de entrega, entre otros tantos que les permita a las empresas dar cuenta de elementos relevantes que los Ileven hacia una ventaja en el mercado con respecto a sus competidores. En conjunto con esto, deberá realizarse una investigación de los clientes con la finalidad de conocer la tendencia de consumo y sus necesidades futuras, puesto que después de haber analizado lo anterior, se obtiene información que permitirá dar paso al diseño y desarrollo de la propuesta de valor.

Una vez analizadas las tendencias del mercado de acuerdo al ramo en donde se desempeña la empresa y las preferencias del posible target de mercado, será importante, cuando sea necesario, considerar lo que sugieren los modelos analizados (e.g., Kline, \& Rosenberg, 1986; Marquis, 1969; Rothwell, 1994; Rothwell, \& Zegveld, 1985) puesto que convienen en realizar investigación de tipo técnica y/o experimental principalmente, que permita justificar la innovación a implementar, dado que, para materializar la innovación se procedería al uso de una matriz QFD (Despliegue de la Función de la Calidad, por sus siglas en inglés Quality Function Deployment), misma que funciona como metodología para interpretar las necesidades del cliente con respecto a la propuesta de valor y es una herramienta clave en la mejora de la calidad en los productos (Gutiérrez, Gutiérrez, Garibay, \& Díaz 2014).

En este sentido, la matriz mencionada, permite encontrar los elementos clave de la propuesta de valor que maximizan la satisfacción del cliente de acuerdo a lo planteado en sus necesidades y crear la ventaja competitiva con respecto a las 
empresas rivales, diseñando y desarrollando la propuesta de valor para su lanzamiento en el mercado.

En este mismo contexto, es de suma importancia enfatizar que se pretende que la innovación se realice de manera continua en las empresas como parte del propio emprendimiento, por consiguiente es necesaria la utilización e implementación de una última herramienta como lo es el Mapa de Ruta Tecnológico (MRT), puesto que por un lado, ayuda a identificar lo que es posible realizar, es decir, permite planear innovaciones futuras y dirigir los esfuerzos de innovación a partir del análisis de las tendencias del mercado (utilizando los procesos de benchmarking e investigando a clientes potenciales) y los problemas o necesidades que deben ser cubiertos en el mediano y largo plazo; y por otro lado analizar las necesidades tecnológicas para llevar a cabo el desarrollo de la propuesta de valor en conjunto a programas de investigación técnica con respecto de la nueva innovación (Faulkner, 1996; Kappel, 2001; Ahmed, Shepherd, Ramos \& Ramos, 2012).

\section{CONCLUSIONES}

Para las pequeñas y medianas empresas (PYME) y de acuerdo a diversos autores (e.g., Freel, 1998; García \& Martínez, 2009; Genatios \& Lafuente, 2004; Heijs, 2001; Moreno, 2013; OCDE, 2006; Pedroza, \& Sánchez 2005; Fernández \& Rodeiro-Pazos, 2006; Zan, 2000) la implementación de un proceso de innovación tiende a ser más complejo debido a la falta de recursos necesarios para llevarlo a cabo, son diversas las limitantes que enfrentan dentro y fuera estas organizaciones, desde el tamaño de la misma, hasta la falta especifica de recursos humanos técnicamente calificados, lo que a su vez, genera problemas en otras áreas como administración (e.g., falta de tiempo dedicado al desarrollo de ventajas competitivas) y mercadotecnia (e.g., falta de análisis del mercado y consciencia del entorno competitivo) .

Por tanto, es importante seguir el proceso en este orden de acuerdo a la propuesta aquí señalada, debido a que es fundamental conocer las tendencias del mercado así como las necesidades de los clientes antes de tomar la decisión de 
Aproximación de una propuesta metodológica para el emprendimiento e implementación de la innovación en PYMES

lanzar un producto o desarrollar una investigación por lo costoso que pueda llegar a ser para una PYME (Benchmarking y QFD), así mismo, es insoslayable realizar un análisis y evaluación financiera del proyecto de innovación, de esta manera, se evita la utilización de recursos de manera ineficiente.

La realización de esta propuesta podría facilitar para las PYMES el acercamiento a un proceso de innovación, y al mismo tiempo se podría contribuir a la solución de los problemas que puedan presentarse en otras áreas (e.g., administración, mercadotecnia), destacando que la innovación se haga parte de la cultura empresarial y de los procesos de planeación de la PYME, lo cual se puede facilitar con el uso del MRT.

También es recomendable impulsar desde las instituciones de gobierno, políticas públicas que incentiven la innovación en las PYMES y posibiliten su supervivencia y desarrollo. De igual forma, es importante impulsar programas educativos dirigidos a los pequeños y medianos empresarios para su formación en políticas y metodologías para la innovación, tratando de hacer conciencia sobre la importancia que tiene la innovación en el desarrollo y fortalecimeinto de las pequeñas y medianas empresas. Finalmente, se puede decir que innovar es emprender, para crear nuevos derroteros que permitan que las PYMES creen valor y oportunidades de desarrollo para la sociedad en su conjunto.

\section{REFERENCIAS BIBLIOGRÁFICAS}

Afuach, Alan (1999), La dinámica de la innovación organizacional: un nuevo enfoque para lograr ventaja competitiva. OXFORD University Press. México.

Ahmed, Pervalz K, Shepherd, Charles D, Ramos, Leticia y Ramos, Claudia. (2012), Administración de la innovación. Pearson Education. México.

Aranda, Heriberto, De la Fuente, Martha Leticia y Becerra, María Nieves (2010), Propuesta metodológica para evaluar la gestión de la innovación tecnológica (GIT) en pequeñas y medianas empresas (PYMES). Revista Mexicana de Agronegocios. 14(26), México, (Pp. 226-238).

Arraut, Luis Carlos (2008), La innovación de tipo organizacional en las empresas manufactureras de catargena de indias. Semestre Económico. 11(22), Colombia, (Pp. 185-203).

Battini, Pierre (1994), Innovar para ganar. Limusa. México. 
Comisión Europea (1995), Libro verde de la innovación. Comisión Europea. Bélgica.

Dinacyt (2003), El proceso de la innovación en la industria uruguaya. Resultados de la Encuesta de Actividades de Innovación (1998-2002). Ministerio de Educación y Cultura, Dirección Nacional de Ciencia, Tecnología e Innovación. Uruguay.

Escorsa, Pere y Valls, Jaume (2003), Tecnología e innovación en la empresa. Universidad Politécnica de Catalunya. España.

Escorsa, Pere y Valls, Jaume (2005), Tecnología e innovación en la empresa (segunda edición). Alfaomega Grupo Editor. México.

Faulkner, Terrence W (1996), Applying 'options thinking' to R\&D valuation. Research Technology Management. 39(3), United Kingdom, (Pp. 50-56).

Fernández, Esteban (1996), Innovación tecnológica y alianzas estratégicas. Factores clave de competencia. Civitas. España.

Fernández, Loreto (1995), La innovación tecnológica: propuesta de un modelo de valoración de proyectos basado en el análisis de triple segmentación y en las particularidades de la empresa gallega. Tesis doctoral. Tesis de Doctorado en Ciencias Económicas y Empresariales. Universidad de Santiago de Compostela, España.

Fernández, Loreto y Rodeiro-Pazos, David (2006), Características de las PYMES gallegas innovadoras frente a las PYMES no innovadoras. Revista Gallega de Economía. 15(2). Extraído de https://www.researchgate.net/profile/Loreto Fernandez2/publication/264962 40 Caracteristicas de las PyMES gallegas innovadoras frente a las $\mathrm{Py}$ MES no innovadoras/links/0c96052f49c37bbb1b000000/Caracteristicasde-las-PyMES-gallegas-innovadoras-frente-a-las-PyMES-noinnovadoras.pdf. Consulta: 13/09/2018.

Franco de Abreu, Emerson, Giuliani, Antonio Carlos, Kassouf, Nadia, y Alves, Dalila (2006), Benchmarking como instrumento dirigido al cliente. Invenio: Revista de investigación académica. 9(17), Argentina, (Pp. 77-94).

Freel, Mark S. (1998), Evolution, Innovation and Learning: Evidence from Case Studies. Entrepreneurship and Regional Development. 10(2), United Kingdom, (Pp. 137-149).

García, Domingo y Martínez, María del Carmen (2009), Innovación y cultura empresarial de las mipyme (micro, pequeña y mediana empresa) Estado de Aguascalientes. Universidad Autónoma de Aguascalientes. México.

García, Juan Vicente y Rodríguez, Javier Alfonso (2010), EI ABC de la innovación. Netbiblo. España.

Genatios, Carlos y Lafuente, Marianela (2004), Ciencia y Tecnología en Venezuela. Ediciones OPSU. Venezuela.

Gutiérrez, Humberto, Gutiérrez, Porfirio, Garibay, Cecilia y Díaz, Lizbeth (2014), Análisis multivariado y QFD como herramientas para escuchar la voz del cliente y mejorar la calidad del servicio. Ingeniare. Revista Chilena de Ingeniería. 22(1), Chile, (Pp. 62-73).

Hamel, Gary (2001), Liderando la revolución. Ediciones Norma. Colombia. 
Heijs, Joost (2001), Justificación de la política tecnológica: un enfoque teórico y metodológico. Documentos de trabajo del IAIF. Universidad Complutense Madrid. Extraído de http://eprints.ucm.es/6758/. Consulta: 08/09/2018.

Instituto Nacional de Estadística y Geografía INEGI (2014), Micro, pequeña, mediana y gran empresa. Extraído de http://internet.contenidos.inegi.org.mx/contenidos/productos/prod serv/cont enidos/espanol/bvinegi/productos/nueva estruc/promo/m pymes ce2014.p df. Consulta: 12/10/2018.

Intxaurburu, Gurutze y Velasco, Eva (2010), El benchmarking aplicado a la gestión de la innovación. Revista de Dirección y Administración de Empresas. (17), España, (Pp. 33-46).

Kappel, Thomas A (2001), Perspectives on roadmaps: how organizations talk about the future. Journal of Product Innovation Management. 18(1), United Kingdom, (Pp. 39-50).

Kline, Stephen. J y Rosenberg, Nathan (1986), "An Overview of Innovation”, en Landan, R., y Rosenberg, N. (Ed.). The Positive Sum Strategy. Harnessing Technology for Economic Growth. Nacional Academic Press. USA.

Marquis, Donald G (1969), The Anatomy of Successful Innovations. Innovation. 1(7), United Kingdom, (Pp. 28-37)

Martín, Jesús (1995), Benchmarking. AECA: Revista de la Asociación Española de Contabilidad y Administración de Empresas. Extraído de http://www.aeca1.org/revistaeca/revista37/37.pdf. Consulta: 08/08/2018.

Moreno, Héctor (2013), La innovación tecnológica como herramienta para el desarrollo de la competitividad en las pymes. Revista Iberoamericana de Contaduría, Economía y Administración: RICEA. Extraído de https://dialnet.unirioja.es/servlet/articulo?codigo $=5825190 . \quad$ Consulta: 08/08/2018.

Organización para la Cooperación y el Desarrollo Económicos OCDE (2006), Manual de Oslo. Guía para la recogida e interpretación de datos sobre innovación (tercera edición). Grupo Tragsa. España.

Ortíz, Alberto (1994), Gerencia Financiera. Un enfoque estratégico. Mc Graw Hill Interamericana, S.A. Colombia.

Pedroza, Álvaro R, Garrido, Luis Edmundo, López, Carlos, Ortiz, Vicente B y Ortiz, Juan Antonio (2013), Innovación y tecnología en la empresa. Claves para adelantarse al futuro. ITESO. México.

Pedroza, Álvaro Rafael y Sánchez, José (2005), Procesos de innovación tecnológica en la pequeña y mediana empresa. Universidad de Guadalajara.México.

Porter, Michael E (1998), Ventaja competitiva. CECSA. México.

Rothwell, Roy (1994), Towards the fifth generation innovation process. International Marketing Review. 11(1), United Kingdom, (Pp. 7-31).

Rothwell, Roy y Zegveld, Walter (1985), Reindustrialization and Technology. Longman. England.

Schumpeter, Joseph A (1934), The Theory of Economic Development. Harvard University Press. USA. 
Schumpeter, Joseph A (1967), Teoría del desenvolvimiento económico. Una investigación sobre ganancias, capital, crédito, interés y ciclo económico (cuarta edición). Fondo de Cultura Económica. México.

Solleiro, José Luis y Castañón, Rosario (2008), Gestión tecnológica: conceptos y prácticas. Plaza y Valdés. México.

Tundidor, Alberto (2017), Como innovar en las pymes. Manual de mejora a través de la innovación (segunda edición). Alfaomega Grupo Editor. México.

Velázquez, Guillermo y Salgado, Josué (2016), Innovación tecnológica: un análisis del crecimiento económico en México (2002-2012: proyección a 2018). Análisis Económico. 31(78), México, (Pp. 145-170).

Wasserman, Moisés (2012), Innovación con conocimiento. Revista Colombiana de Ciencias Pecuarias. 25(4), Colombia, (Pp. 537-538).

Zan, Marta (2000), Redes de innovación. Redes. 7(15), Argentina, (Pp. 139-150). 